\title{
Research Article \\ Fixed Points of Multivalued Maps in Modular Function Spaces
}

\author{
Marwan A. Kutbi and Abdul Latif \\ Department of Mathematics, King Abdulaziz University, P. O. Box 80203, Jeddah 21589, Saudi Arabia \\ Correspondence should be addressed to Abdul Latif, latifmath@yahoo.com \\ Received 7 February 2009; Accepted 14 April 2009 \\ Recommended by Jerzy Jezierski \\ The purpose of this paper is to study the existence of fixed points for contractive-type and \\ nonexpansive-type multivalued maps in the setting of modular function spaces. We also discuss \\ the concept of $w$-modular function and prove fixed point results for weakly-modular contractive \\ maps in modular function spaces. These results extend several similar results proved in metric and \\ Banach spaces settings.
}

Copyright (C) 2009 M. A. Kutbi and A. Latif. This is an open access article distributed under the Creative Commons Attribution License, which permits unrestricted use, distribution, and reproduction in any medium, provided the original work is properly cited.

\section{Introduction and Preliminaries}

The well-known Banach fixed point theorem on complete metric spaces (specifically, each contraction self-map of a complete metric space has a unique fixed point) has been extended and generalized in different directions. For example, see Edelstein [1, 2], Kasahara [3], Rhoades [4], Siddiq and Ansari [5], and others. One of its generalizations is for nonexpansive single-valued maps on certain subsets of a Banach space. Indeed, these fixed points are not necessarily unique. See, for example, Browder [6-8] and Kirk [9]. Fixed point theorems for contractive and nonexpansive multivalued maps have also been established by several authors. Let $H$ denote the Hausdorff metric on the space of all bounded nonempty subsets of a metric space $(X, d)$. A multivalued map $J: X \rightarrow 2^{X}$ (where $2^{X}$ denotes the collection of all nonempty subsets of $X$ ) with bounded subsets as values is called contractive [10] if

$$
H(J(x), J(y)) \leq h d(x, y)
$$

for all $x, y \in X$ and for a fixed number $h \in[0,1)$. If the Lipschitz constant $h=1$, then $J$ is called a multivalued nonexpansive mapping [11]. Nadler [10], Markin [11], Lami-Dozo [12], and others proved fixed point theorems for these maps under certain conditions in the setting of 
metric and Banach spaces. Note that an element $x \in X$ is called a fixed point of a multivalued map $J: X \rightarrow 2^{X}$ if $x \in J(x)$. Among others, without using the concept of the Hausdorff metric, Husain and Tarafdar [13] introduced the notion of a nonexpansive-type multivalued map and proved a fixed point theorem on compact intervals of the real line. Using such type of notions Husain and Latif [14] extended their result to general Banach space setting.

The fixed point results in modular function spaces were given by Khamsi et al. [15]. Even though a metric is not defined, many problems in metric fixed point theory can be reformulated in modular spaces. For instance, fixed point theorems are proved in $[15,16]$ for nonexpansive maps.

In this paper, we define nonexpansive-type and contractive-type multivalued maps in modular function spaces, investigate the existence of fixed points of such mappings, and prove similar results found in [17].

Now, we recall some basic notions and facts about modular spaces as formulated by Kozlowski [18]. For more details the reader may consult $[15,16]$.

Let $\Omega$ be a nonempty set and let $\Sigma$ be a nontrivial $\sigma$-algebra of subsets of $\Omega$. Let $D$ be a $\delta$-ring of subsets of $\Sigma$, such that $E \cap A \in D$ for any $E \in D$ and $A \in \Sigma$.

Let us assume that there exists an increasing sequence of sets $K_{n} \in D$ such that $\Omega=$ $\bigcup K_{n}$. By $\mathcal{\varepsilon}$ we denote the linear space of all simple functions with supports from $D$. By $\mathcal{M}$ we will denote the space of all measurable functions, that is, all functions $f: \Omega \rightarrow \mathbb{R}$ such that there exists a sequence $\left\{g_{n}\right\} \in \mathcal{E},\left|g_{n}\right| \leq|f|$ and $g_{n}(\omega) \rightarrow f(\omega)$ for all $\omega \in \Omega$. By $1_{A}$ we denote the characteristic function of the set $A$.

Definition 1.1. A functional $\rho: \varepsilon \times \Sigma \rightarrow[0, \infty]$ is called a function modular if

$\left(P_{1}\right) \rho(0, E)=0$ for any $E \in \Sigma$,

$\left(P_{2}\right) \rho(f, E) \leq \rho(g, E)$ whenever $|f(\omega)| \leq|g(\omega)|$ for any $\omega \in \Omega, f, g \in \mathcal{E}$ and $E \in \Sigma$,

$\left(P_{3}\right) \rho(f, \cdot): \Sigma \rightarrow[0, \infty]$ is a $\sigma$-subadditive measure for every $f \in \mathcal{E}$,

$\left(P_{4}\right) \rho(\alpha, A) \rightarrow 0$ as $\alpha$ decreases to 0 for every $A \in D$, where $\rho(\alpha, A)=\rho\left(\alpha 1_{A}, A\right)$,

$\left(P_{5}\right)$ if there exists $\alpha>0$ such that $\rho(\alpha, A)=0$, then $\rho(\beta, A)=0$ for every $\beta>0$, and

$\left(P_{6}\right)$ for any $\alpha>0, \rho(\alpha,$.$) is order continuous on D$, that is, $\rho\left(\alpha, A_{n}\right) \rightarrow 0$ if $\left\{A_{n}\right\} \in \mathcal{D}$ and decreases to $\emptyset$.

The definition of $\rho$ is then extended to $f \in \mathcal{M}$ by

$$
\rho(f, E)=\sup \{\rho(g, E) ; g \in \varepsilon,|g(\omega)| \leq|f(\omega)|, \text { for every } \omega \in \Omega\}
$$

For the sake of simplicity we write $\rho(f)$ instead of $\rho(f, \Omega)$.

Definition 1.2. A set $E$ is said to be $\rho$-null if $\rho(\alpha, E)=0$ for every $\alpha>0$. A property $p(w)$ is said to hold $\rho$-almost everywhere ( $\rho$-a.e.) if the set $\{w \in \Omega: p(w)$ does not hold $\}$ is $\rho$-null.

Definition 1.3. A modular function $\rho$ is called $\sigma$-finite if there exists an increasing sequence of sets $K_{n} \in P$ such that $0<\rho\left(K_{n}\right)<\infty$ and $\Omega=\bigcup K_{n}$. It is easy to see that the functional 
$\rho: \mathcal{M} \rightarrow[0, \infty]$ is a modular and satisfies the following properties:

(i) $\rho(f)=0$ if and only if $f=0 \rho$-a.e.,

(ii) $\rho(\alpha f)=\rho(f)$ for every scalar $\alpha$ with $|\alpha|=1$ and $f \in \mathcal{M}$, and

(iii) $\rho(\alpha f+\beta g) \leq \rho(f)+\rho(g)$ if $\alpha+\beta=1, \alpha \geq 0, \beta \geq 0$ and $f, g \in \mathcal{M}$.

In addition, if the following property is satisfied,

(iii)' $\rho(\alpha f+\beta g) \leq \alpha \rho(f)+\beta \rho(g)$ if $\alpha+\beta=1, \alpha \geq 0, \beta \geq 0$ and, $f, g \in \mathcal{M}$,

we say that $\rho$ is a convex modular.

The modular $\rho$ defines a corresponding modular space, that is, the vector space $L_{\rho}$ given by

$$
L_{\rho}=\{f \in \mathcal{M} ; \rho(\lambda f) \longrightarrow 0 \text { as } \lambda \longrightarrow 0\} .
$$

When $\rho$ is convex, the formula

$$
\|f\|_{p}=\inf \left\{\alpha>0 ; \rho\left(\frac{f}{\alpha}\right) \leq 1\right\}
$$

defines a norm in the modular space $L_{\rho}$ which is frequently called the Luxemburg norm. We can also consider the space

$$
E_{\rho}=\left\{f \in \mathcal{M} ; \rho\left(\alpha f, A_{n}\right) \rightarrow 0 \text { as } n \rightarrow \infty \text { for every } A_{n} \in \Sigma \text { that decreases to } \emptyset \text { and } \alpha>0\right\}
$$

Definition 1.4. A function modular is said to satisfy the $\Delta_{2}$-condition if $\sup _{n \geq 1} \rho\left(2 f_{n}, D_{k}\right) \rightarrow$ 0 as $k \rightarrow \infty$ whenever $\left\{f_{n}\right\}_{n \geq 1} \subset \mathcal{M}, D_{k} \in \Sigma$ decreases to $\emptyset$ and $\sup _{n \geq 1} \rho\left(f_{n}, D_{k}\right) \rightarrow 0$ as $k \rightarrow \infty$.

We know from [18] that $E_{\rho}=L_{\rho}$ when $\rho$ satisfies the $\Delta_{2}$-condition.

Definition 1.5. A function modular is said to satisfy the $\Delta_{2}$-type condition if there exists $K>0$ such that for any $f \in L_{\rho}$ we have $\rho(2 f) \leq K \rho(f)$.

In general, $\Delta_{2}$-type condition and $\Delta_{2}$-condition are not equivalent, even though it is obvious that $\Delta_{2}$-type condition implies $\Delta_{2}$-condition on the modular space $L_{\rho}$.

Definition 1.6. Let $\succeq_{\rho}$ be a modular space.

(1) The sequence $\left\{f_{n}\right\} \subset L_{\rho}$ is said to be $\rho$-convergent to $f \in L_{\rho}$ if $\rho\left(f_{n}-f\right) \rightarrow 0$ as $n \rightarrow \infty$.

(2) The sequence $\left\{f_{n}\right\} \subset L_{\rho}$ is said to be $\rho$-a.e. convergent to $f \in L_{\rho}$ if the set $\{\omega \in$ $\left.\Omega ; f_{n}(\omega) \nrightarrow f(\omega)\right\}$ is $\rho$-null.

(3) The sequence $\left\{f_{n}\right\} \subset L_{\rho}$ is said to be $\rho$-Cauchy if $\rho\left(f_{n}-f_{m}\right) \rightarrow 0$ as $n$ and $m$ go to $\infty$.

(4) A subset $C$ of $L_{\rho}$ is called $\rho$-closed if the $\rho$-limit of a $\rho$-convergent sequence of $C$ always belongs to $C$. 
(5) A subset $C$ of $L_{\rho}$ is called $\rho$-a.e. closed if the $\rho$-a.e. limit of a $\rho$-a.e. convergent sequence of $C$ always belongs to $C$.

(6) A subset $C$ of $L_{\rho}$ is called $\rho$-a.e. compact if every sequence in $C$ has a $\rho$-a.e. convergent subsequence in $C$.

(7) A subset $C$ of $L_{\rho}$ is called $\rho$-bounded if

$$
\delta_{\rho}(C)=\sup \{\rho(f-g) ; f, g \in C\}<\infty
$$

We recall two basic results (see [15]) in the theory of modular spaces.

(i) If there exists a number $\alpha>0$ such that $\rho\left(\alpha\left(f_{n}-f\right)\right) \rightarrow 0$, then there exists a subsequence $\left\{g_{n}\right\}$ of $\left\{f_{n}\right\}$ such that $g_{n} \rightarrow f \rho$-a.e.

(ii) (Lebesgue's Theorem) If $f_{n}, f \in \mathcal{M}, f_{n} \rightarrow f \rho$-a.e. and there exists a function $g \in E_{\rho}$ such that $\left|f_{n}\right| \leq|g| \rho$-a.e. for all $n$, then $\left\|f_{n}-f\right\|_{p} \rightarrow 0$.

We know, by $[15,16]$ that under $\Delta_{2}$-condition the norm convergence and modular convergence are equivalent, which implies that the norm and modular convergence are also the same when we deal with the $\Delta_{2}$-type condition. In the sequel we will assume that the modular function $\rho$ is convex and satisfies the $\Delta_{2}$-type condition.

Definition 1.7. Let $\rho$ be as aforementioned. We define a growth function $\omega$ by

$$
\omega(t)=\sup \left\{\frac{\rho(t f)}{\rho(f)}, f \in L_{\rho} \backslash\{0\}\right\} \quad \forall 0 \leq t<\infty .
$$

We have the following:

Lemma 1.8 (see [19]). Let $\rho$ be as aforementioned. Then the growth function $\omega$ has the following properties:

(1) $\omega(t)<\infty, \forall t \in[0, \infty)$,

(2) $\omega:[0, \infty) \rightarrow[0, \infty)$ is a convex, strictly increasing function. So, it is continuous,

(3) $\omega(\alpha \beta) \leq \omega(\alpha) \omega(\beta) ; \forall \alpha, \beta \in[0, \infty)$,

(4) $\omega^{-1}(\alpha) \omega^{-1}(\beta) \leq \omega^{-1}(\alpha \beta) ; \forall \alpha, \beta \in[0, \infty)$, where $\omega^{-1}$ is the function inverse of $\omega$.

The following lemma shows that the growth function can be used to give an upper bound for the norm of a function.

Lemma 1.9 (see [19]). Let $\rho$ be a convex function modular satisfying the $\Delta_{2}$-type condition. Then

$$
\|f\|_{p} \leq \frac{1}{\omega^{-1}(1 / \rho(f))} \text { whenever } f \in L_{\rho}
$$

The next lemma will be of major interest throughout this work. 
Lemma 1.10 (see [16]). Let $\rho$ be a function modular satisfying the $\Delta_{2}$-condition and let $\left\{f_{n}\right\}$ be a sequence in $L_{\rho}$ such that $f_{n} \stackrel{\rho-a . e}{\rightarrow} f \in L_{\rho}$, and there exists $k>1$ such that $\sup _{n} \rho\left(k\left(f_{n}-f\right)\right)<\infty$. Then,

$$
\liminf _{n \rightarrow \infty} \rho\left(f_{n}-g\right)=\liminf _{n \rightarrow \infty} \rho\left(f_{n}-f\right)+\rho(f-g) \quad \forall g \in L_{\rho}
$$

Moreover, one has

$$
\rho(f) \leq \liminf _{n \rightarrow \infty} \rho\left(f_{n}\right)
$$

\section{Fixed Points of Contractive-Type and Nonexpansive-Type Maps}

In the sequel we assume that $\rho$ is a convex, $\sigma$-finite modular function satisfying the $\Delta_{2}$-type condition, and $C$ is a nonempty $\rho$-bounded subset of the modular function space $L_{\rho}$. We denote that $\mathcal{C}(C)$ is a collection of all nonempty $\rho$-closed subsets of $C$, and $\mathcal{K}(C)$ is a collection of all nonempty $\rho$-compact subsets of $C$.

We say that a multivalued map $T: C \rightarrow 2^{C}$ is $\rho$-contractive-type if there exists $k \in$ $(0,1)$ such that for any $f, g \in C$ and for any $F \in T(f)$, there exists $G \in T(g)$ such that

$$
\rho(F-G) \leq k \rho(f-g)
$$

and $\rho$-nonexpansive-type if for any $f, g \in C$ and for any $F \in T(f)$, there exists $G \in T(g)$ such that

$$
\rho(F-G) \leq \rho(f-g)
$$

We have the following fixed point theorem (for which a similar result may be found in [17]).

Theorem 2.1. Let $C$ be a nonempty $\rho$-closed subset of the modular function space $L_{\rho}$. Then any $T: C \rightarrow \mathcal{C}(C) \rho$-contractive-type map has a fixed point, that is, there exists $f \in C$ such that $f \in T(f)$.

Proof. Let $f_{0} \in C$. Without loss of generality, assume that $f_{0}$ is not a fixed point of $T$. Then there exists $f_{1} \in T\left(f_{0}\right)$ such that $f_{1} \neq f_{0}$. Hence $\rho\left(f_{0}, f_{1}\right)>0$. Since $T$ is $\rho$-contractive-type, then there exists $f_{2} \in T\left(f_{1}\right)$ such that

$$
\rho\left(f_{1}-f_{2}\right) \leq k \rho\left(f_{0}-f_{1}\right)
$$

By induction, one can easily construct a sequence $\left\{f_{n}\right\} \in C$ such that $f_{n+1} \in T\left(f_{n}\right)$ and

$$
\rho\left(f_{n+1}-f_{n}\right) \leq k \rho\left(f_{n}-f_{n-1}\right),
$$


for any $n \geq 1$. In particular we have

$$
\rho\left(f_{n+1}-f_{n}\right) \leq k^{n} \rho\left(f_{1}-f_{0}\right) .
$$

Without loss of generality, we may assume $\rho\left(f_{n+1}, f_{n}\right) \neq 0$, otherwise $f_{n}$ is a fixed point of $T$. Hence

$$
\frac{1}{k^{n} \rho\left(f_{1}-f_{0}\right)} \leq \frac{1}{\rho\left(f_{n+1}-f_{n}\right)}
$$

Using Lemma 1.9, we get

$$
\left\|f_{n+1}-f_{n}\right\|_{\rho} \leq \frac{1}{\omega^{-1}\left(1 / \rho\left(f_{n+1}-f_{n}\right)\right)} .
$$

Using the properties of $\omega(t)$, we get

$$
\omega^{-1}\left(\frac{1}{k^{n} \rho\left(f_{1}-f_{0}\right)}\right) \leq \omega^{-1}\left(\frac{1}{\rho\left(f_{n+1}-f_{n}\right)}\right) .
$$

So

$$
\omega^{-1}\left(\frac{1}{k}\right)^{n} \omega^{-1}\left(\frac{1}{\rho\left(f_{1}-f_{0}\right)}\right) \leq \omega^{-1}\left(\frac{1}{\rho\left(f_{n+1}-f_{n}\right)}\right)
$$

which implies

$$
\left\|f_{n+1}-f_{n}\right\|_{\rho} \leq \frac{1}{\omega^{-1}(1 / k)^{n} \omega^{-1}\left(1 / \rho\left(f_{1}-f_{0}\right)\right)}
$$

Since $\omega(1)=1$ and $k<1$, then $1<\omega^{-1}(1 / k)$. This forces $\left\{f_{n}\right\}$ to be $\|\cdot\|_{\rho}$-Cauchy. Hence the sequence $\left\{f_{n}\right\}\|\cdot\|_{\rho}$-converges to some $f \in L_{\rho}$. Since $\rho$ satisfies the $\Delta_{2}$-condition, then $\left\{f_{n}\right\} \rho$ converges to $f$. Since $C$ is $\rho$-closed, then $f \in C$. Let us prove that $f$ is indeed a fixed point of $T$. Since $T$ is a $\rho$-contractive-type mapping, then for any $n \geq 1$, there exists $F_{n} \in T(f)$ such that

$$
\rho\left(f_{n+1}-F_{n}\right) \leq k \rho\left(f_{n}-f\right) .
$$

Hence $\left\{\rho\left(f_{n+1}-F_{n}\right)\right\}$ converges to 0 . Since $\rho$ satisfies the $\Delta_{2}$-condition, we have $\left\{\left\|f_{n+1}-F_{n}\right\|_{\rho}\right\}$ converges to 0 . Since $\left\{f_{n}\right\}\|\cdot\|_{\rho}$-converges to $f$, then $\left\{F_{n}\right\}\|\cdot\|_{\rho}$-converges to $f$. Hence $\left\{F_{n}\right\} \rho$ converges to $f$. Since $T(f)$ is $\rho$-closed and $\left\{F_{n}\right\} \in T(f)$, we get $f \in T(f)$.

Remark 2.2. Consider the multivalued map $T_{A}(f)=A$, where $A$ is a nonempty $\rho$-closed subset of $C$. Then it is easy to show that $T_{A}$ is a $\rho$-contractive-type map. The set of all fixed 
point of $T_{A}$ is exactly the set $A$. In particular, $\rho$-contractive-type maps may not have a unique fixed point.

As an application of the above theorem, we have the following result.

Proposition 2.3. Let $C$ be a $\rho$-closed convex subset of the modular function space $L_{\rho}$. Let $T: C \rightarrow$ $\mathcal{C}(C)$ be $\rho$-nonexpansive-type map. Then there exists an approximate fixed points sequence $\left\{f_{n}\right\}$ in $C$, that is, for any $n \geq 1$ there exists $F_{n} \in T\left(f_{n}\right)$ such that

$$
\lim _{n \rightarrow \infty} \rho\left(f_{n}-F_{n}\right)=0
$$

In particular one has $\lim _{n \rightarrow \infty} \operatorname{dist}_{\rho}\left(f_{n}, T\left(f_{n}\right)\right)=0$, where

$$
\operatorname{dist}_{\rho}\left(f_{n}, T\left(f_{n}\right)\right)=\inf \left\{\rho\left(f_{n}-g\right) ; g \in T\left(f_{n}\right)\right\}
$$

Proof. Let $\lambda \in(0,1)$ and let $f_{0}$ be a fixed point in $C$. For each $f \in C$, define a map

$$
T_{\lambda}(f)=\lambda f_{0}+(1-\lambda) T(f)=\left\{\lambda f_{0}+(1-\lambda) g ; g \in T(f)\right\}
$$

Note that $T_{\lambda}(f)$ is nonempty and $\rho$-closed subset of $C$ because $T(f)$ is $\rho$-closed and $C$ is convex. Since $T$ is a $\rho$-nonexpansive-type map, for each $f, g \in C$ and for any $F \in T(f)$, there exists $G \in T(g)$ such that

$$
\rho(F-G) \leq \rho(f-g)
$$

Since $\rho$ is convex we get

$$
\rho\left(\left(\lambda f_{0}+(1-\lambda) F\right)-\left(\lambda f_{0}+(1-\lambda) G\right)\right)=\rho((1-\lambda)(F-G)) \leq(1-\lambda) \rho(F-G),
$$

which implies

$$
\rho\left(\left(\lambda f_{0}+(1-\lambda) F\right)-\left(\lambda f_{0}+(1-\lambda) G\right)\right) \leq(1-\lambda) \rho(f-g)
$$

In other words, the map $T_{\lambda}$ is a $\rho$-contractive-type. Theorem 2.1 implies the existence of a fixed point $f_{\lambda}$ of $T_{\lambda}$, thus there exists $F_{\lambda} \in T\left(f_{\lambda}\right)$ such that

$$
f_{\lambda}=\lambda f_{0}+(1-\lambda) F_{\lambda}
$$

In particular, we have

$$
\rho\left(f_{\lambda}-F_{\curlywedge}\right)=\rho \lambda\left(f_{0}-F_{\lambda}\right) \leq \lambda \rho\left(f_{0}-F_{\lambda}\right) \leq \lambda \delta_{\rho}(C),
$$


where $\delta_{\rho}(C)=\sup _{f, g \in C} \rho(f-g)$ is the $\rho$-diameter of $C$. Note that since $C$ is $\rho$-bounded, then $\delta_{\rho}(C)<\infty$. If we choose $\lambda=1 / n$, for $n \geq 1$ and write $f_{n}=f_{\lambda_{n}}$ and $F_{n}=F_{\lambda_{n}}$, we get

$$
\rho\left(f_{n}-F_{n}\right) \leq \frac{\delta_{\rho}(C)}{n}
$$

for any $n \geq 1$, which implies $\lim _{n \rightarrow \infty} \rho\left(f_{n}-F_{n}\right)=0$.

Using the above result, we are now ready to prove the main fixed point result for $\rho$-nonexpansive-type multivalued maps.

Theorem 2.4. Let $C$ be a nonempty $\rho$-closed convex subset of the modular function space $L_{\rho}$. Assume that $C$ is $\rho$-a.e. compact. Then each $\rho$-nonexpansive-type map $T: C \rightarrow \mathcal{K}(C)$ has a fixed point.

Proof. Proposition 2.3 ensures the existence of a sequence $\left\{f_{n}\right\}$ in $C$ and a sequence $\left\{F_{n}\right\}$ such that $F_{n} \in T\left(f_{n}\right)$ and $\lim _{n \rightarrow \infty} \rho\left(f_{n}-F_{n}\right)=0$. Without loss of generality we may assume that $\left\{f_{n}\right\} \rho$-a.e. converges to $f \in C$ and $\left\{F_{n}\right\} \rho$-a.e. converges to $F \in C$. Lemma 1.10 implies

$$
\rho(f-F) \leq \liminf _{n \rightarrow \infty} \rho\left(f_{n}-F_{n}\right)=0 .
$$

Hence $f=F$. Since $T$ is a $\rho$-nonexpansive-type map, then there exists a sequence $\left\{G_{n}\right\} \in T(f)$ such that

$$
\rho\left(F_{n}-G_{n}\right) \leq \rho\left(f_{n}-f\right),
$$

for all $n \geq 1$. Since $T(f)$ is $\rho$-compact, we may assume that $\left\{G_{n}\right\}$ is $\rho$-convergent to some $h \in T(f)$. Lemma 1.10 implies

$$
\liminf _{n \rightarrow \infty} \rho\left(f_{n}-f\right)+\rho(f-h)=\liminf _{n \rightarrow \infty} \rho\left(f_{n}-h\right) .
$$

Since $\rho$ satisfies the $\Delta_{2}$-condition, then

$$
\begin{aligned}
\liminf _{n \rightarrow \infty} \rho\left(f_{n}-h\right) & =\liminf _{n \rightarrow \infty} \rho\left(f_{n}-F_{n}+F_{n}-G_{n}+G_{n}-h\right) \\
& =\liminf _{n \rightarrow \infty} \rho\left(F_{n}-G_{n}\right)
\end{aligned}
$$

(see, [20]). Since $\rho\left(F_{n}-G_{n}\right) \leq \rho\left(f_{n}-f\right)$, we get

$$
\liminf _{n \rightarrow \infty} \rho\left(f_{n}-h\right) \leq \liminf _{n \rightarrow \infty} \rho\left(f_{n}-f\right),
$$

which implies

$$
\liminf _{n \rightarrow \infty} \rho\left(f_{n}-f\right)+\rho(f-h) \leq \liminf _{n \rightarrow \infty} \rho\left(f_{n}-f\right) .
$$


Fixed Point Theory and Applications

Hence $\rho(f-h)=0$ or $f=h$. Hence $f \in T(f)$; that is, $f$ is a fixed point of $T$.

Proposition 2.3 and Theorem 2.4 are also hold if we assume that $C$ is starshaped instead of Convex. (A set $C$ is called starshaped if there exists $f_{0} \in C$ such that $\lambda f_{0}-(1-\lambda) f \in$ $C$ provided $f \in C$ and $\lambda \in[0,1]$.)

\section{Fixed Points of $w$-Contractive-Type Maps}

In [21] the authors introduced the concept of $w$-distance in metric spaces which they connected to the existence of fixed point of single and multivalued maps (see also [22]). Similarly we extend their definition and results to modular spaces. Indeed let $\rho$ be a convex, $\sigma$-finite modular function. A function $p: L_{\rho} \times L_{\rho} \rightarrow[0, \infty)$ is called $w$-modular on the modular function space $L_{\rho}$ if the following are satisfied:

(1) $p(f, g) \leq p(f, h)+p(h, g)$ for any $f, g, h \in L_{\rho}$;

(2) for any $f \in L_{\rho}, p(f, \cdot): L_{\rho} \rightarrow[0, \infty)$ is lower semicontinuous; that is, if $\left\{g_{n}\right\} \rho$ converges to $g$, then

$$
p(f, g) \leq \liminf _{n \rightarrow \infty} p\left(f, g_{n}\right)
$$

(3) for any $\varepsilon>0$, there exists $\delta>0$ such that $p(f, g) \leq \delta$ and $p(f, h) \leq \delta$ imply $\rho(g, h) \leq$ $\varepsilon$.

As it was done in [21], we need the following technical lemma.

Lemma 3.1. Let $p(\cdot, \cdot)$ be w-modular on the modular function space $L_{\rho}$. Let $\left\{f_{n}\right\}$ and $\left\{g_{n}\right\}$ be sequences in $L_{\rho}$, and let $\left\{\alpha_{n}\right\}$ and $\left\{\beta_{n}\right\}$ be sequences in $[0, \infty)$ converging to 0 , and $f, g, h \in L_{\rho}$. Then the following hold:

(1) if $p\left(f_{n}, g\right) \leq \alpha_{n}$ and $p\left(f_{n}, h\right) \leq \beta_{n}$, for all $n \geq 1$, then $g=h$; in particular if $p(f, g)=0$ and $p(f, h)=0$, then $g=h$;

(2) if $p\left(f_{n}, g_{n}\right) \leq \alpha_{n}$ and $p\left(f_{n}, h\right) \leq \beta_{n}$, for any $n \geq 1$, then $\left\{g_{n}\right\} \rho$-converges to $h$;

(3) if $p\left(f_{n}, f_{m}\right) \leq \alpha_{n}$ for any $n, m \geq 1$ with $m>n$, then $\left\{f_{n}\right\}$ is a $\rho$-Cauchy sequence;

(4) if $p\left(g, f_{n}\right) \leq \alpha_{n}$ for any $n \geq 1$, then $\left\{f_{n}\right\}$ is a $\rho$-Cauchy sequence.

The proof is easy and similar to the one given in [21]. Now we are ready to give the first fixed point result in this setting. Let $C$ be a nonempty $\rho$-closed subset of the modular function space $L_{\rho}$. We say that a multivalued map $T: C \rightarrow \mathcal{C}(C)$ is weakly $\rho$-contractivetype map if there exists $w$-modular $p(\cdot, \cdot)$ on $L_{\rho}$ and $k \in[0,1)$ such that for any $f, g \in C$ and any $F \in T(f)$, there exists $G \in T(g)$ such that $p(F, G) \leq k p(f, g)$.

Theorem 3.2. Let $C$ be a nonempty $\rho$-closed subset of the modular function space $L_{\rho}$. Then each weakly $\rho$-contractive-type map $T: C \rightarrow \mathcal{C}(C)$ has a fixed point $f \in C$, and $p(f, f)=0$. 
Proof. Let $p(\cdot, \cdot)$ be a $w$-modular and $k \in[0,1)$ associated to $T$, that is, for any $f, g \in C$ and any $F \in T(f)$, there exists $G \in T(g)$ such that $p(F, G) \leq k p(f, g)$. Fix $f_{0} \in C$ and $f_{1} \in T\left(f_{0}\right)$. By induction one can construct a sequence $\left\{f_{n}\right\}$ such that $f_{n+1} \in T\left(f_{n}\right)$ and

$$
p\left(f_{n}, f_{n+1}\right) \leq k p\left(f_{n-1}, f_{n}\right),
$$

for every $n \geq 1$. In particular we have $p\left(f_{n}, f_{n+1}\right) \leq k^{n} p\left(f_{0}, f_{1}\right)$, for every $n \geq 1$. Using the properties of $p(\cdot, \cdot)$, we get

$$
p\left(f_{n}, f_{n+h}\right) \leq \frac{k^{n}}{1-k} p\left(f_{0}, f_{1}\right)
$$

for any $n, h \geq 1$. Lemma 3.1 implies that the sequence $\left\{f_{n}\right\}$ is $\rho$-Cauchy. Hence $\left\{f_{n}\right\} \rho$ converges to some $f \in C$. Using the lower semicontinuity of $p$, we get

$$
p\left(f_{n}, f\right) \leq \liminf _{n \rightarrow \infty} p\left(f_{n}, f_{n+h}\right) \leq \frac{k^{n}}{1-k} p\left(f_{0}, f_{1}\right),
$$

for any $n \geq 1$. Since $f_{n} \in T\left(f_{n-1}\right)$ and $T$ is weakly $\rho$-contractive-type map, there exists $g_{n} \in$ $T(f)$ such that

$$
p\left(f_{n}, g_{n}\right) \leq k p\left(f_{n-1}, f\right) \leq \frac{k^{n}}{1-k} p\left(f_{0}, f_{1}\right)
$$

for any $n \geq 2$. Lemma 3.1 implies that $\left\{g_{n}\right\} \rho$-converges to $f$ as well. Since $T(f)$ is $\rho$-closed, then $f \in T(f)$, that is, $f$ is a fixed point of $T$. Let us complete the proof by showing that $p(f, f)=0$. Since $f \in T(f)$, there exists $h_{1} \in T(f)$ such that $p\left(f, h_{1}\right) \leq k p(f, f)$. By induction we can construct a sequence $\left\{h_{n}\right\}$ in $C$ such that $h_{n+1} \in T\left(h_{n}\right)$ and $p\left(f, h_{n+1}\right) \leq k p\left(f, h_{n}\right)$, for any $n \geq 1$. So we have $p\left(f, h_{n}\right) \leq k^{n} p(f, f)$, for any $n \geq 1$. Lemma 3.1 implies that $\left\{h_{n}\right\}$ is $\rho$-Cauchy. Hence $\left\{h_{n}\right\} \rho$ - converges to some $h \in C$. Using the lower semicontinuity of $p(\cdot, \cdot)$ we get

$$
p(f, h) \leq \liminf _{n \rightarrow \infty} p\left(f, h_{n}\right) \leq 0
$$

Hence $p(f, h)=0$. Then for any $n \geq 1$, we have

$$
p\left(f_{n}, h\right) \leq p\left(f_{n}, f\right)+p(f, h) \leq \frac{k^{n}}{1-k} p\left(f_{0}, f_{1}\right)
$$

Lemma 3.1 implies $f=h$, or $p(f, f)=0$.

Note that in the proof above we did not use the $\Delta_{2}$-condition. The reason behind is that $p(\cdot, \cdot)$ satisfies the triangle inequality. If $T$ is single valued, then we have little more information about the fixed point. Indeed, let $C$ be a nonempty $\rho$-closed subset of the modular function space $L_{\rho}$. The map $T: C \rightarrow C$ is called a weakly $\rho$-contractive type map if there exists $w$-modular $p(\cdot, \cdot)$ on $L_{\rho}$ and $k \in[0,1)$ such that for any $f, g \in C ; p(T(f), T(g)) \leq$ $k p(f, g)$. 
Theorem 3.3. Let $C$ be a nonempty $\rho$-closed subset of the modular function space $L_{\rho}$. Then each weakly $\rho$-contractive type map $T: C \rightarrow C$ has a unique fixed point $f \in C$, and $p(f, f)=0$.

Proof. Theorem 3.2 ensures the existence of a fixed point $f \in C$, that is, $T(f)=f$ and $p(f, f)=$ 0 . Let us show that $f$ is the only fixed point of $T$. Assume that $h \in C$ is another fixed point of $T$. Then we must have $p(f, h)=0$. Combining this with $p(f, f)=0$, Lemma 3.1 implies $f=h$.

Similar extensions of the results as found in [21-23] may be proved in our setting.

\section{Acknowledgments}

The authors thank the referees for their valuable comments and suggestions. The authors would also like to thank Professor M.A. Khamsi for productive discussion and cooperation regarding this work.

\section{References}

[1] M. Edelstein, "An extension of Banach's contraction principle," Proceedings of the American Mathematical Society, vol. 12, no. 1, pp. 7-10, 1961.

[2] M. Edelstein, "On fixed and periodic points under contractive mappings," Journal of the London Mathematical Society, vol. 37, pp. 74-79, 1962.

[3] S. Kasahara, "On some generalizations of the Banach contraction theorem," Publications of the Research Institute for Mathematical Sciences, vol. 12, no. 2, pp. 427-437, 1976.

[4] B. E. Rhoades, "A comparison of various definitions of contractive mappings," Transactions of the American Mathematical Society, vol. 226, pp. 257-290, 1977.

[5] A. H. Siddiqi and Q. H. Ansari, "An iterative method for generalized variational inequalities," Mathematica Japonica, vol. 34, no. 3, pp. 475-481, 1989.

[6] F. E. Browder, "On a theorem of Beurling and Livingston," Canadian Journal of Mathematics, vol. 17, pp. 367-372, 1965.

[7] F. E. Browder, "Fixed-point theorems for noncompact mappings in Hilbert space," Proceedings of the National Academy of Sciences of the United States of America, vol. 53, no. 6, pp. 1272-1276, 1965.

[8] F. E. Browder, "Nonexpansive nonlinear operators in a Banach space," Proceedings of the National Academy of Sciences of the United States of America, vol. 54, no. 4, pp. 1041-1044, 1965.

[9] W. A. Kirk, "A fixed point theorem for mappings which do not increase distances," The American Mathematical Monthly, vol. 72, no. 9, pp. 1004-1006, 1965.

[10] S. B. Nadler Jr., "Multi-valued contraction mappings," Pacific Journal of Mathematics, vol. 30, no. 2, pp. 475-488, 1969.

[11] J. T. Markin, "A fixed point theorem for set valued mappings," Bulletin of the American Mathematical Society, vol. 74, pp. 639-640, 1968.

[12] E. Lami Dozo, "Multivalued nonexpansive mappings and Opial's condition," Proceedings of the American Mathematical Society, vol. 38, no. 2, pp. 286-292, 1973.

[13] T. Husain and E. Tarafdar, "Fixed point theorems for multivalued mappings of nonexpansive type," Yokohama Mathematical Journal, vol. 28, no. 1-2, pp. 1-6, 1980.

[14] T. Husain and A. Latif, "Fixed points of multivalued nonexpansive maps," International Journal of Mathematics and Mathematical Sciences, vol. 14, no. 3, pp. 421-430, 1991.

[15] M. A. Khamsi, W. M. Kozłowski, and S. Reich, "Fixed point theory in modular function spaces," Nonlinear Analysis: Theory, Methods E Applications, vol. 14, no. 11, pp. 935-953, 1990.

[16] M. A. Khamsi, "Fixed point theory in modular function spaces," in Recent Advances on Metric Fixed Point Theory (Seville, 1995), vol. 48 of Ciencias, pp. 31-57, Universidad de Sevilla, Seville, Spain, 1996.

[17] S. Dhompongsa, T. Domínguez Benavides, A. Kaewcharoen, and B. Panyanak, "Fixed point theorems for multivalued mappings in modular function spaces," Scientiae Mathematicae Japonicae, vol. 63, no. 2, pp. 161-169, 2006.

[18] W. M. Kozłowski, Modular Function Spaces, vol. 122 of Monographs and Textbooks in Pure and Applied Mathematics, Marcel Dekker, New York, NY, USA, 1988. 
[19] T. Dominguez Benavides, M. A. Khamsi, and S. Samadi, "Uniformly Lipschitzian mappings in modular function spaces," Nonlinear Analysis: Theory, Methods E Applications, vol. 46, no. 2, pp. 267278, 2001.

[20] T. Dominguez-Benavides, M. A. Khamsi, and S. Samadi, "Asymptotically regular mappings in modular function spaces," Scientiae Mathematicae Japonicae, vol. 53, no. 2, pp. 295-304, 2001.

[21] O. Kada, T. Suzuki, and W. Takahashi, "Nonconvex minimization theorems and fixed point theorems in complete metric spaces," Mathematica Japonica, vol. 44, no. 2, pp. 381-391, 1996.

[22] T. Suzuki and W. Takahashi, "Fixed point theorems and characterizations of metric completeness," Topological Methods in Nonlinear Analysis, vol. 8, no. 2, pp. 371-382, 1996.

[23] T. Suzuki, "Generalized distance and existence theorems in complete metric spaces," Journal of Mathematical Analysis and Applications, vol. 253, no. 2, pp. 440-458, 2001. 